Originalien

Nervenarzt 2021 · 92:1172-1178 https://doi.org/10.1007/s00115-020-01013-9 Angenommen: 6. September 2020 Online publiziert: 14 . Oktober 2020 ๑) Der/die Autor(en) 2020

\section{Zusatzmaterial online}

Die Onlineversion dieses Beitrags (https:// doi.org/10.1007/s00115-020-01013-9) enthält weitere Infomaterialien. Beitrag und Zusatzmaterial stehen Ihnen auf www. springermedizin.de zur Verfügung. Bitte geben Sie dort den Beitragstitel in die Suche ein, das Zusatzmaterial finden Sie beim Beitrag unter „Ergänzende Inhalte“.

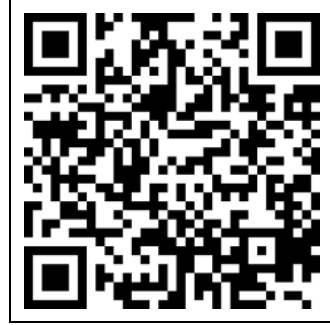

\section{Hintergrund und Fragestellung}

In Deutschland wird aktuell eine Überschuldungsquote von $10 \%$ angenommen, sodass schätzungsweise über 6,9 Mio. Menschen über 18 Jahre in Deutschland bestehende Schulden nicht begleichen können, da kein Vermögen und keine zukünftigen Einnahme absehbar sind [18]. Die Altersstruktur der überschuldeten Personen zeigt, dass die

Stefanie Schreiter ${ }^{1}$ Sascha Heidrich ${ }^{1}$ Andreas Heinz $z^{1,2}$. Wulf Rössler ${ }^{1,3}$. Reinhard Michael Krausz ${ }^{4}$ Meryam Schouler-Ocak ${ }^{1,2} \cdot$ Felix Bermpohl $^{1,2}$. Stefan Gutwinski ${ }^{1,2}$

' Department of Psychiatry and Psychotherapy, Charité Campus Mitte, Charité - Universitätsmedizin Berlin, corporate member of Freie Universität Berlin, Humboldt-Universität zu Berlin, and Berlin Institute of Health, Berlin, Deutschland

${ }^{2}$ Psychiatrische Universitätsklinik der Charité im St. Hedwig Krankenhaus, Berlin, Deutschland ${ }^{3}$ Klinik für Psychiatrie und Psychotherapie, Psychiatrische Universitätsklinik, Universität Zürich, Zürich, Schweiz

${ }^{4}$ Institute of Mental Health and Department of Psychiatry, University of British Columbia (UBC),

Vancouver, Kanada

\title{
Schulden, Kredite und unbezahlte Rechnungen unter Nutzer*Innen teilstationärer und stationärer psychiatrischer Behandlung in Berlin
}

Gruppe der 30- bis 39-Jährigen mit jeder 5. Person die höchste Verschuldungsquote aufweist [18]. Wie das Statistische Bundesamt in einer Untersuchung von 559 der insgesamt rund 1450 Schuldnerberatungsstellen in Deutschland feststellte, lagen Erkrankungen, Sucht oder Unfälle mit 15,8\% nach der Arbeitslosigkeit auf Rang 2 der Hauptursachen für eine Überschuldung [19]. Laut den Ergebnissen einer Metaanalyse ist die Odds Ratio bezüglich des Vorliegens einer psychischen Erkrankung unter verschuldeten Personen gegenüber Personen ohne Schulden 3-fach erhöht [16]. Bisherige Studien zeigten vor allem $\mathrm{Zu}-$ sammenhänge zwischen Überschuldung und seelischen Störungen („common mental disorders") [13-16] und globaler psychischer Belastung (ermittelt mit dem General Health Questionnaire; [7, 16]) sowie Drogengebrauch, ,problem drinking" und Rauchen $[9,13,16]$. Die eindeutigste Datenlage zu psychiatrischen Diagnosen findet sich bei depressiven Störungen und zeigt kontrolliert für verschiedene konfundierende Faktoren und in standardisierten Erhebungen einen signifikanten Zusammenhang zum
Faktor Schulden [13, 15, 16]. Ähnliche Befunde zeigen sich in einzelnen Studien auch bei Angsterkrankungen [4, 13] und psychotischen Störungen [9]. Zudem ist das Suizidrisiko im Zusammenhang mit Schulden erhöht: Die Rate von Suizidversuchen ist bei überschuldeten Personen 5-mal so hoch wie in der Allgemeinbevölkerung [7], bei Personen mit einem durchgeführten Suizid ist das Risiko für das Vorliegen von Schulden 8-fach erhöht [16].

Hinsichtlich des Zusammenhangs zwischen objektiven Variablen der Überschuldung und dem Vorliegen einer psychischen Erkrankung fanden einige Studien Zusammenhänge zwischen der objektiven Höhe und Anzahl der Schulden und der Schwere der Gesundheitsprobleme $[9,13,16]$. Allerdings bleiben die Mechanismen des Zusammenwirkens zwischen Schulden und Gesundheitsproblemen bzw. psychischer Erkrankung ungeklärt, wobei Studien auf verschiedene psychologische Aspekte der Verarbeitung hindeuten, wie beispielsweise (mal)adaptive Copingstrategien [8], Sorgen und Stress 
bezüglich Schulden [4] oder Gefühle von Hoffnungslosigkeit [12].

Bisherige Studien untersuchten den Zusammenhang zwischen psychischen Erkrankungen und Verschuldung in Bevölkerungsstichproben und spezifischen Populationen wie Studenten, ältere Menschen oder Klienten von Schuldenberatungen [16]. Untersuchungen unter psychiatrischen Patient ${ }^{\star}$ Innen sind uns bisher aus Deutschland nicht bekannt. Angesichts der Erreichbarkeit möglicher Angebote für Menschen mit psychischen Erkrankungen und finanzieller Belastung erscheint die nähere Betrachtung des Vorliegens von Verschuldung in einer Stichprobe von bereits bestehenden NutzerInnen des Gesundheitssystems sinnvoll. Wir führten daher eine explorative querschnittliche Untersuchung hinsichtlich des Vorliegens finanzieller Belastungen und assoziierter soziodemografischer wie klinischer Faktoren unter Patient ${ }^{\star}$ Innen im Rahmen einer (teil-)stationären psychiatrischen Behandlung in Berlin durch.

\section{Studiendesign und Unter- suchungsmethoden}

\section{Durchführung und Stichprobe}

Zwischen dem 15.03.und dem 15.09.2016 führten wir eine Befragung in Form eines strukturierten Interviews $\mathrm{zu}$ Schulden und anderen soziodemografischen und krankheitsbezogenen Variablen unter allen Patient ${ }^{\star}$ Innen der Psychiatrischen
Universitätsklinik der Charité im St. Hedwig Krankenhaus durch, die sich in (teil-)stationärer psychiatrischer Behandlung befanden.

Eine ausführliche Beschreibung des Studiendesigns wurde an anderer Stelle veröffentlicht [17].

Die Ethikkommission der Charité Universitätsmedizin Berlin bewertete die Studie positiv (Nummer: EA1/291/15). Eine schriftliche Einwilligung vor der Studienteilnahme wurde eingeholt. Die Befragung dauerte durchschnittlich eine Stunde; 11 Interviews (2\%) erfolgten mithilfe eines professionellen Sprach- und Kulturvermittlers.

Von den $1251 \mathrm{im}$ Studienzeitraum zu einer (teil-)stationären psychiatrischen Behandlung aufgenommenen Personen nahmen $540(43,2 \%)$ an der Befragung teil. $328(26,2 \%)$ lehnten eine Teilnahme ab und 383 (30,6\%) konnten zur Befragung nicht erreicht werden (siehe [17]). 488 Teilnehmer*Innen gaben Auskunft $\mathrm{zu}$ bestehenden Schulden, Krediten und offenen Rechnungen. Nach einem Gruppenvergleich zwischen Personen mit und ohne Schulden erfolgte zum Ausschluss von Personen mit geringen Schulden eine Subgruppenanalyse von Personen mit Schulden $>1000 €$ vs. keine Schulden.

\section{Messinstrumente}

Soziodemografische Variablen, das Vorliegen von Schulden und die Schuldenhöhe sowie das Nutzungsverhalten innerhalb des Versorgungssystems und Va- riablen zur Verschuldungssituation wurden im Rahmen des strukturierten Interviews erfasst. Bei fehlenden oder unklaren Daten wurden diese mit der Dokumentation der Sozialarbeiter ${ }^{\star}$ Innen der Klinik abgeglichen. Psychiatrische Diagnosen basierten auf den Entlassungsdiagnosen nach den Kriterien des ICD10 [6].

Die Erfassung der Wohnsituation basierte auf der Hauptwohnform in den letzten 30 Tagen vor der Klinikaufnahme.

\section{Statistische Analysen}

Statistische Analysen erfolgten mittels SPSS 19 [20]. Deskriptive Analysen erfolgten mit den entsprechenden statistischen Parametern (Mittelwert, Standardabweichung, Median, Quartile). Gruppenvergleiche erfolgten entsprechend den vorliegenden Daten entweder mittels $\chi^{2}$ - bzw. T-Test; im Falle nicht normalverteilter Daten wurde der MannWhitney-Test durchgeführt. Signifikante Faktoren der Gruppenvergleiche wurden als Faktoren in ein binär logistisches Regressionsmodell eingeführt, wobei im 1. Modell das Vorliegen von Schulden, Krediten oder offenen Rechnungen und im 2. Modell das Vorliegen von Schulden über $1000 €$ als abhängige Variable diente. Es erfolgte eine Anpassung des $p$-Werts mittels Bonferroni-Methode.

Hier steht eine Anzeige. 


\section{Ergebnisse}

Von den 488 Teilnehmer ${ }^{\star}$ Innen, die Auskunft über ihre finanzielle Situation gaben, wiesen 269 (55,1\%) Schulden, Kredite oder offene Rechnungen auf. Unter den Teilnehmer* Innen, die Auskunft zur Kredit- oder Schuldenhöhe machten $(n=215)$, wiesen $14,4 \%$ Schulden oder Kredite in der Höhe von unter $1000 €$ auf, $47,0 \%$ zwischen 1000 und 9999€, 36,3\% zwischen 10.000 und $99.999 €$ und $2,3 \%$ über $100.000 €$ (• Tab. 1).

Hinsichtlich soziodemografischer und klinischer Faktoren waren Teilnehmer ${ }^{\star}$ Innen mit Schulden, Krediten oder offenen Rechnungen signifikant häufiger jüngeren Alters, männlichen Geschlechts und in Wohnungslosigkeit; sie lebten signifikant häufiger von Sozialleistungen, wiesen signifikant häufiger mehrere psychiatrische Komorbiditäten auf und waren signifikant häufiger an folgenden psychischen Störungen erkrankt: organische psychische Störungen, schädlicher Gebrauch einer Substanz und Substanzabhängigkeiten sowie Persönlichkeitsstörungen. Sie wiesen signifikant seltener affektive Störungen und Intelligenzminderungen auf (•Tab. 2). Im Vergleich zwischen Teilnehmer ${ }^{\star}$ Innen ohne Schulden oder Krediten und Teilnehmer Innen mit Schulden oder Krediten über $1000 €$ wiesen Teilnehmer ${ }^{\star}$ Innen mit Schulden oder Krediten über $1000 €$ neben den zuvor beschriebenen Gruppenunterschieden zusätzlich signifikant seltener psychotische Erkrankungen auf (s. Zusatzmaterial online eTabelle 1).

Nach Einführung signifikanter Variablen der Gruppenvergleiche in ein binär logistisches Regressionsmodell zur Vorhersage des Vorliegens von Schulden, Krediten oder offenen Rechnungen ergaben sich ein jüngeres Alter $(\mathrm{OR}=0,98$; Bonferroni-korrigiertes $p=0,036)$ und das Vorliegen einer Substanzabhängigkeit $(\mathrm{OR}=2,41$; Bonferroni-korrigiertes $p<0,001)$ als signifikant assoziierte Faktoren (•Tab. 3). Im 2. Modell zur Vorhersage des Vorliegens von Schulden oder Krediten über $1000 €$ ergaben sich ebenfalls ein jüngeres Alter $(\mathrm{OR}=0,98$; Bonferroni-korrigiertes $p=0,018$ ) und das Vorliegen einer Substanzabhängigkeit $(\mathrm{OR}=2,41$; Bonferroni-korrigiertes

Nervenarzt 2021 · 92:1172-1178 https://doi.org/10.1007/s00115-020-01013-9

(c) Der/die Autor(en) 2020

S. Schreiter · S. Heidrich · A. Heinz · W. Rössler · R. M. Krausz · M. Schouler-Ocak · F. Bermpohl · S. Gutwinski

\section{Schulden, Kredite und unbezahlte Rechnungen unter Nutzer*Innen teilstationärer und stationärer psychiatrischer Behandlung in Berlin}

\section{Zusammenfassung}

Hintergrund. Bisherige Studien der Allgemeinbevölkerung weisen auf eine Assoziation zwischen psychischen Erkrankungen und verschiedenen Formen finanzieller Schwierigkeiten wie Verschuldung hin. Ziel der Arbeit. Untersuchung der finanziellen Belastungen und assoziierter Faktoren bei Patient ${ }^{*}$ Innen in (teil-)stationärer psychiatrischer Behandlung.

Material und Methoden. Insgesamt 488 Teilnehmer*Innen einer querschnittlichen Patientenbefragung mittels eines strukturierten Interviews zu soziodemografischen sowie klinischen Variablen in (teil)stationärer psychiatrischer Behandlung in der psychiatrischen Universitätsklinik der Charité im St. Hedwig, zuständig für einen spezifischen Versorgungsbereich in Berlin, gaben Auskunft zu finanziellen Belastungen. Ergebnisse. Insgesamt 269 (55,1\%) Teilnehmer*Innen wiesen Schulden, Kredite oder offene Rechnungen auf. Unter den Teilnehmer*Innen, die Auskunft zur Kredit- oder Schuldenhöhe machten $(n=215)$, wies der größte Teil (47,0\%) Schulden oder Kredite in der Höhe zwischen 1000 und $9999 €$ auf, gefolgt von 36,3\% mit Schulden/Krediten zwischen 10.000 und $99.999 €$. In den Regressionsmodellen hinsichtlich des Vorliegens von Schulden erwiesen sich ein jüngeres Alter und das Vorliegen einer Substanzabhängigkeit als signifikant assoziierte Faktoren. 22,3\% der Befragten wiesen Schulden in Höhe $>10.000 €$ auf und lebten von Sozialleistung, sodass eine Überschuldung angenommen werden könnte.

Diskussion. Finanzielle Belastungen und bestehende Schulden sollten im psychiatrischen Bereich stärker in der Praxis erfragt und beachtet werden. Geeignete Unterstützungsformen sollten entwickelt und evaluiert werden.

Schlüsselwörter

Schulden · Wohnungslosigkeit · Armut .

Psychiatrie · Psychische Erkrankung

\section{Debts, loans and unpaid bills among day patients and inpatients in psychiatric care in Berlin, Germany}

\section{Abstract}

Background. Previous studies among the general population indicated an association between mental illnesses and different forms of financial difficulties, such as indebtedness. Objective. Investigation of the financial burden and associated factors among inpatients and day clinic patients in psychiatric care. Material and methods. A total of 488 patients in psychiatric care in the catchment area of the Psychiatric University Hospital Charité at St. Hedwig Hospital participated in a crosssectional patient survey carried out with a structured interview regarding financial burden, sociodemographic and clinical variables.

Results. Of the participants 269 (55.1\%) showed outstanding debts, loans or unpaid bills. Among the participants who were willing to give information about the amount of debts, the majority (47.0\%) had debts between $1000 €$ and $9999 €$ and $36.3 \%$ between $10,000 €$ and $99,999 €$. In the binary regression models, younger age and substance use disorders were factors significantly associated with outstanding debts. Of the participants $22.3 \%$ had outstanding debts $>10,000 €$ and were depending on social welfare, so that indebtedness could be assumed. Conclusion. Financial burdens and outstanding debts among patients in psychiatric care should be inquired about and considered more intensively in practice. Suitable models of support need to be developed and evaluated.

Keywords

Debts · Homelessness · Mental illness . Poverty · Psychiatry 
Tab. 1 Teilnehmer*Innen mit Schulden:

Verteilung nach Schuldenhöhe

\begin{tabular}{ll}
$\begin{array}{l}\text { Schuldenhöhe } \\
(\boldsymbol{n}=\mathbf{2 1 5})\end{array}$ & $\begin{array}{l}\text { Anzahl Pa- } \\
\text { tient*Innen }\end{array}$ \\
\hline$<1000 €$ & $31(14,4 \%)$ \\
\hline $1000-9999 €$ & $101(47,0 \%)$ \\
\hline $10.000-99.999 €$ & $78(36,3 \%)$ \\
$>100.000 €$ & $5(2,3 \%)$
\end{tabular}

$p=0,040)$ als signifikant assoziierte Faktoren (• Tab. 3).

Von der gesamten Stichprobe lebten $71,0 \%$ von Sozialleistungen $(n=448)$; Teilnehmer^Innen mit Schulden, Krediten oder offenen Rechnungen lebten zu 78,7\% von Sozialleistungen $(n=448)$; bei einer Höhe von über $10.000 €(n=60)$ waren es $80,0 \%$.

\section{Diskussion}

Dies ist die erste Erhebung finanzieller Belastungen unter Nutzer*Innen des psychiatrischen Gesundheitsversorgungssystems in Deutschland. In unserer Studie wiesen mehr als die Hälfte der Patient ${ }^{\star}$ Innen $(55,1 \%)$ Schulden, Kredite oder offene Rechnungen auf. Die Mehrzahl (47,0\%) zwischen 1000 und $9999 €$ gefolgt von $36,3 \%$ mit Schulden, Krediten oder offenen Rechnungen in Höhe von 10.000 bis $99.999 €$. Da $80,0 \%$ der Personen mit Schulden, Krediten oder offenen Rechnungen in einer Summe von über $10.000 €$ von Sozialleistungen leben, ist mindestens hier das Vorliegen einer Überschuldung sehr wahrscheinlich, wobei die Bewertung einer Überschuldung immer eine Einzelfallentscheidung ist und auch von anderen Faktoren abhängt (Alter, Zahl der Gläubiger, finanzielle Situation anderer Haushaltsmitglieder, mögliches Einkommen etc.; [5]). Zukünftige Erhebungen sollten daher interindividuelle Unterschiede insbesondere in Bezug auf das Haushaltseinkommen stärker berücksichtigen.

Davon ausgehend würde es bedeuten, dass eine Überschuldung 22,3\% der Befragten, die Angaben zur Schuldenhöhe machten, betrifft. Damit läge die Zahl deutlich höher als die Überschuldungsrate der deutschen Allgemeinbevölkerung von $10 \%$ [18] und würde somit bisherige Studienergebnisse bestätigen, die eine deutliche Assoziation zwischen psychischen Erkrankungen und Verschuldung belegen [16]. Auch in Bezug auf die Wohnsituation zeigt sich, dass der Faktor Wohnungslosigkeit mit dem Vorkommen von Schulden verbunden ist (75,0\% aller wohnungslosen Teilnehmer wiesen Schulden auf, vgl. eigene Wohnung: 51,7\%). Eine kürzlich veröffentlichte longitudinale Studie weist auf einen Zusammenhang zwischen psychischer Gesundheit und Sorgen um die Bezahlbarkeit von Wohnraum („housing affordability stress“) hin [3]. Zukünftige Erhebungen sollten daher interindividuelle Unterschiede insbesondere in Bezug auf diese Faktoren, wie beispielsweise das Haushaltseinkommen, stärker berücksichtigen.

Die Ergebnisse verdeutlichen die Notwendigkeit einer holistischen Blickweise auf psychische Erkrankungen und soziale Stressoren wie Verschuldung und Armut sowie die Notwendigkeit der Evaluierung und Implementierung geeigneter sozialer Unterstützungen im Gesundheitsbereich psychischer Erkrankungen bzw. die Stärkung der Rolle der sozialen Arbeit. Beispiele hierfür sind in Großbritannien zu finden, wo die Regierung während der letzten Rezession zusätzliche Therapien für Menschen unter ökonomischer Belastung finanzierte und Schuldenberatung in Gesundheitseinrichtungen unterstützte [10].

Ergebnisse der Regressionsmodelle wiesen dabei vor allem auf Substanzabhängigkeiten und ein jüngeres Alter als prädiktive Faktoren für das Vorliegen von Schulden hin. Eine Implementierung geeigneter Unterstützungsformen vor allem im Suchthilfebereich erscheint daher sinnvoll. Umgekehrt stellt sich Frage, ob Angebote zur Beratung hinsichtlich psychischer Erkrankungen und insbesondere Substanzgebrauch z.B. in Einrichtungen wie Schuldnerberatungen implementiert und evaluiert werden sollten. Das jüngere Alter deckt sich mit Erhebungen der Allgemeinbevölkerung, in der ebenfalls hinsichtlich der Altersstruktur eher Menschen jüngeren Alters von Überschuldung betroffen sind [18]. Insbesondere die frühe Unterstützung junger Menschen mit psychischen
Erkrankungen hinsichtlich finanzieller Belastungen scheint daher besonders wichtig, um einer Marginalisierung vorzubeugen.

Ein mögliches Instrument zur Prävention von Schulden und Unterstützung finanzieller Belange stellt in Deutschland die gesetzliche Betreuung dar. Allerdings fanden sich keine signifikanten Gruppenunterschiede zwischen Teilnehmer ${ }^{\star}$ Innen mit und ohne Schulden, offenen Rechnungen oder Krediten hinsichtlich des Vorliegens einer gesetzlichen Betreuung. Inwieweit jedoch möglicherweise insbesondere eine gesetzliche Betreuung in Fällen bereits bestehender finanzieller Schwierigkeiten eingesetzt wurde, wurde im Rahmen dieser Studie nicht untersucht.

Bezüglich möglicher Ursachen der Überschuldung zeigte sich in einer anderen Querschnittserhebung unter überschuldeten Personen in Schweden, dass überschuldete Teilnehmer ${ }^{\star}$ Innen mit einer psychischen Erkrankung jünger waren und häufiger maladaptive Copingstrategien nutzten [8]. Dies wiederum spricht für die Möglichkeit, durch psychotherapeutische Interventionen Einfluss auf das subjektive Erleben finanzieller Belastung zu nehmen, was z. B. in Projekten in Großbritannien durch die Schaffung von Therapien für Menschen unter finanzieller Belastung und Schuldenberatung in Gesundheitseinrichtungen aufgegriffen wurde [10].

An dieser Stelle soll auch auf den Aspekt der finanziellen Teilhabe und Verfügung über eigene finanzielle Mittel hingewiesen werden. In Anbetracht der kürzlichen Einführung des Bundesteilhabegesetzes, das wiederum eine Trennung von beispielsweise Wohn- und anderen Unterstützungsleistungen vorsieht und somit auch mehr Verantwortung und Herausforderung hinsichtlich der Finanzierung der verschiedenen Leistungsträger seitens der Nutzer ${ }^{\star}$ Innen birgt, sollten finanzielle Schwierigkeiten stärker berücksichtigt werden [11]. Dabei sollten Schulden sowohl als mögliche Folgen einer psychischen Erkrankung in Betracht gezogen werden sowie auch als Risikofaktor für das Entstehen psychischer Störungen, z. B. von Suchterkrankungen. 
Tab. 2 Soziodemografische und klinische Gruppenunterschiede zwischen Teilnehmer*Innen mit und ohne Schulden, Kredite oder offenen Rechnungen

\begin{tabular}{|c|c|c|c|}
\hline $\begin{array}{l}\text { Schulden, Kredite oder unbezahlte Rech- } \\
\text { nungen } \\
(n=488)^{\mathrm{a}}\end{array}$ & $\begin{array}{l}\text { Vorliegende Schulden, Kredite } \\
\text { oder unbezahlte Rechnungen }\end{array}$ & $\begin{array}{l}\text { Keine Schulden, Kredite oder } \\
\text { unbezahlte Rechnungen }\end{array}$ & Statistik \\
\hline Anzahl Teilnehmer*Innen & $269(55,1 \%)$ & $219(44,9 \%)$ & - \\
\hline Anzahl männliche Teilnehmer ${ }^{b}$ & $175(65,1 \%)$ & $109(50,0 \%)$ & $X^{2}(1)=11,22 ; p=0,001$ \\
\hline Alter $(M \pm S D)$ & $39,89( \pm 11,84)$ & $44,51( \pm 17,05)$ & $\mathrm{T}=3,40 ; p=0,001$ \\
\hline Bildungsjahre (Median [IQR]) & $13,5(11,5-16,0)$ & $15,0(12-0-17,0)$ & $Z=-1,89 ; p=0,059$ \\
\hline Wohnstatus $^{\mathrm{c}}$ & & & $X^{2}(3)=10,69 ; p=0,014$ \\
\hline Eigene Wohnung & $152(57,4 \%)$ & $142(65,4 \%)$ & - \\
\hline Gesundheitsbezogene Einrichtungen & $45(17,0 \%)$ & $36(16,5 \%)$ & - \\
\hline Wohnungslos & $42(15,8 \%)$ & $14(6,5 \%)$ & - \\
\hline Bei Freunden/Familie & $26(9,8 \%)$ & $25(11,5 \%)$ & - \\
\hline Einkommen & & & $X^{2}(2)=24,64 ; p<0,001$ \\
\hline $\begin{array}{l}\text { Gehalt (Voll- oder Teilzeitarbeit, Ausbildung, } \\
\text { BAföG-Bezug, Erspartes) }\end{array}$ & $46(18,5 \%)$ & $49(24,6 \%)$ & - \\
\hline Sozialleistungen & $196(78,7 \%)$ & $122(61,3 \%)$ & - \\
\hline Altersrente & $7(2,8 \%)$ & $28(14,1 \%)$ & - \\
\hline Verheiratet oder in fester Partnerschaft & $76(28,5 \%)$ & $57(26,3 \%)$ & $X^{2}(1)=0,29 ; p=0,332$ \\
\hline Im Ausland geboren & $65(24,3 \%)$ & $45(20,6 \%)$ & $\mathrm{X}^{2}(1)=0,90 ; p=0,210$ \\
\hline \multicolumn{4}{|l|}{ Psychische Erkrankungen } \\
\hline Organische psychische Störungen & $8(3,0 \%)$ & $16(7,3 \%)$ & $X^{2}(1)=4,85 ; p=0,023$ \\
\hline Psychotische Erkrankungen & $60(22,3 \%)$ & $62(28,3 \%)$ & $\mathrm{X}^{2}(1)=2,32 ; p=0,078$ \\
\hline Substanzabhängigkeit (außer Nikotin) & $160(59,5 \%)$ & $64(29,2 \%)$ & $X^{2}(1)=44,50 ; p<0,001$ \\
\hline $\begin{array}{l}\text { Schädlicher Gebrauch einer Substanz (außer } \\
\text { Nikotin) }\end{array}$ & $63(23,4 \%)$ & $32(14,6 \%)$ & $X^{2}(1)=5,97 ; p=0,009$ \\
\hline Affektive Störungen & $72(26,8 \%)$ & $87(39,7 \%)$ & $X^{2}(1)=9,23 ; p=0,002$ \\
\hline Angststörungen & $10(3,7 \%)$ & $9(4,1 \%)$ & $X^{2}(1)=0,05 ; p=0,502$ \\
\hline Persönlichkeitsstörungen & $64(23,8 \%)$ & $35(16,0 \%)$ & $X^{2}(1)=4,55 ; p=0,021$ \\
\hline Intelligenzminderungen & $3(1,1 \%)$ & $9(4,1 \%)$ & $X^{2}(1)=4,51 ; p=0,033$ \\
\hline $\begin{array}{l}\text { Anzahl psychiatrischer Diagnosen nach ICD-10 } \\
\text { (außer Nikotin) (Median [IQR]) }\end{array}$ & $1,6(1-2)$ & $1,4(1-2)$ & $Z=-2,68 ; p=0,007$ \\
\hline $\begin{array}{l}\text { Alter der ersten psychiatrischen Behandlung } \\
\text { (Median [IQR]) }\end{array}$ & $26,0( \pm 12,57)$ & $26,5( \pm 15,90)$ & $Z=-0,66 ; p=0,512$ \\
\hline $\begin{array}{l}\text { Anzahl der Teilnehmer*Innen mit einem Suizid- } \\
\text { versuch in der Vorgeschichte }\end{array}$ & $91(33,8 \%)$ & $60(27,4 \%)$ & $X^{2}(1)=2,34 ; p=0,076$ \\
\hline Vorliegen einer gesetzlichen Betreuung & $62(23,1 \%)$ & $49(22,5 \%)$ & $X^{2}(1)=0,03 ; p=0,476$ \\
\hline \multicolumn{4}{|c|}{$\begin{array}{l}n=52 \text { fehlend oder ausgeschlossen } \\
{ }^{b} \text { Ein Teilnehmer mit dem Geschlecht Transgender wurde bei Geschlecht nicht berücksichtigt } \\
{ }^{c} \text { Der Wohnstatus wurde in vier Gruppen eingeteilt: wohnungslose Teilnehmer*Innen (einschließlich Menschen, die direkt auf der Straße oder in sonstigen } \\
\text { Verschlägen leben, in Notunterkünften oder sonstigen Einrichtungen der Wohnungslosenhilfe unterkommen, in Flüchtlingsheimen oder Frauenhäusern); } \\
\text { Teilnehmer*Innen in einer eigenen Wohnung oder Wohneigentum; Teilnehmer*Innen in Einrichtungen des Gesundheitswesens bzw. der Eingliederungshilfe } \\
\text { (therapeutische Wohngemeinschaften, Trägerwohnungen des betreuten Einzelwohnens, Übergangswohnheime, Krankenheime etc.); Teilnehmer*Innen, } \\
\text { die bei Freunden, Bekannten oder Familie lebten } \\
\text { IQR Interquartilsabstand }\end{array}$} \\
\hline
\end{tabular}

\section{Limitationen}

Die Angabe der Schuldenhöhe wurde nicht, z.B. anhand von Kontoauszügen oder Angehörigen überprüft, sodass diese allein auf Aussagen der Teilnehmer*innen beruhen und möglicherweise nicht präzise sind. Allerdings wurden unvollständige oder unklare Angaben mit Dokumentationen der Sozialarbeiter ${ }^{\star}$ Innen der Klinik abgeglichen. Zudem ist denkbar, dass Patient ${ }^{\star}$ Innen mit Schulden bspw. aus Gründen von Scham eine Teilnahme ablehnten.

Weitere longitudinale Studien - möglichst interventionellen Charakters - wä- ren notwendig, um die Planung der Versorgung effektiver zu gestalten. Die Generalisierbarkeit der Ergebnisse ist eingeschränkt, da es sich um Nutzer ${ }^{\star}$ Innen eines spezifischen Behandlungssettings eines Bezirks (Wedding, Moabit, Tiergarten) in Berlin handelt, zudem gibt es ein Stichprobenbias, da in der Studie u. a. 
Tab. 3 Prädiktoren für Schulden, Kredite oder unbezahlte Rechnungen: multivariable binäre logistische Regressionsmodelle

\begin{tabular}{|c|c|c|c|c|}
\hline \multirow[b]{2}{*}{ Variablen } & \multicolumn{2}{|c|}{$\begin{array}{l}\text { Schulden, Kredite oder } \\
\text { unbezahlte Rechnungen } \\
(n=441)\end{array}$} & \multirow{2}{*}{$\begin{array}{l}\text { Schulden } \\
>1000 € \\
\text { Adjustierte OR } \\
(95 \%-K I)\end{array}$} & \multirow[b]{2}{*}{$p^{* *}$} \\
\hline & $\begin{array}{l}\text { Adjustierte OR } \\
\text { (95\%-KI) }\end{array}$ & $p^{*}$ & & \\
\hline Geschlecht (männlich vs. weiblich) & $0,63(0,41-0,98)$ & 0,084 & $0,69(0,42-1,12)$ & 0,260 \\
\hline Alter & $0,98(0,96-1,00)$ & 0,036 & $0,97(0,96-0,99)$ & 0,018 \\
\hline \multicolumn{5}{|l|}{ Wohnstatus $^{a}$} \\
\hline Eigene Wohnung & 1 & - & 1 & - \\
\hline Wohnungslos & $1,81(0,86-3,80)$ & 0,232 & $1,67(0,78-3,56)$ & 0,372 \\
\hline Gesundheitsbezogene Einrichtungen & $0,83(0,46-1,49)$ & 1 & $1,05(0,56-1,99)$ & 1 \\
\hline Bei Freunden/Familie & $0,86(0,42-1,77)$ & 1 & $0,95(0,44-2,06)$ & 1 \\
\hline $\begin{array}{l}\text { Einkommen (Gehalt/Altersrente vs. } \\
\text { Sozialleistungen) }\end{array}$ & $0,66(0,41-1,08)$ & 0,198 & $0,60(0,34-1,04)$ & 0,132 \\
\hline Organische psychische Störungen & $0,76(0,27-2,20)$ & 1 & $0,45(0,12-1,71)$ & 0,486 \\
\hline Psychotische Erkrankungen & - & - & $0,64(0,29-1,38)$ & 0,506 \\
\hline $\begin{array}{l}\text { Schädlicher Gebrauch einer Substanz } \\
\text { (außer Nikotin) }\end{array}$ & $1,02(0,57-1,82)$ & 1 & $1,02(0,54-1,94)$ & 1 \\
\hline Substanzabhängigkeit (außer Nikotin) & $2,41(1,48-3,92)$ & $<0,001$ & $2,22(1,14-4,34)$ & 0,040 \\
\hline Affektive Störungen & $0,89(0,51-1,55)$ & 1 & $0,88(0,40-1,94)$ & 1 \\
\hline Persönlichkeitsstörungen & $1,07(0,55-2,08)$ & 1 & $0,87(0,38-2,04)$ & 1 \\
\hline Intelligenzminderungen & $0,24(0,05-1,09)$ & 0,128 & $0,17(0,03-1,04)$ & 0,112 \\
\hline Anzahl an psychiatrischen Diagnosen & $1,43(0,90-2,27)$ & 0,264 & $1,54(0,87-2,72)$ & 0,278 \\
\hline \multicolumn{5}{|c|}{$\begin{array}{l}\text { Der adjustierte } p \text {-Wert wurde mittels Bonferroni-Methode berechnet ( } p \times 2 \text { für die soziodemografi- } \\
\text { schen und klinischen Faktoren) } \\
\text { aIm Vergleich mit eigenerWohnung; derWohnstatus wurde in vier Gruppen eingeteilt:wohnungslose } \\
\text { Teilnehmer*Innen (einschließlich Menschen, die direkt auf der Straße oder in sonstigen Verschlägen } \\
\text { leben, in Notunterkünften oder sonstigen Einrichtungen der Wohnungslosenhilfe unterkommen, } \\
\text { in Flüchtlingsheimen oder Frauenhäusern); Teilnehmer*Innen in einer eigenen Wohnung oder } \\
\text { Wohneigentum; Teilnehmer*Innen in Einrichtungen des Gesundheitswesens bzW. der Eingliede- } \\
\text { rungshilfe (therapeutische Wohngemeinschaften, Trägerwohnungen des betreuten Einzelwohnens, } \\
\text { Übergangswohnheime, Krankenheime etc.); Teilnehmer*Innen, die bei Freunden, Bekannten oder } \\
\text { Familie lebten } \\
\text { *Signifikanz des Modells: } p<0,001 \\
\text { ** Signifikanz des Modells: } p<0,001\end{array}$} \\
\hline
\end{tabular}

signifikant häufiger Personen mit Abhängigkeitserkrankungen, männlichen $\mathrm{Ge}$ schlechts und jüngeren Alters teilgenommen hatten (siehe Beschreibung der Population in Schreiter et al. [17]). Berlin wies 2019 eine verhältnismäßig hohe Arbeitslosenquote mit $11,4 \%$ auf, allerdings ist diese vergleichbar mit Quoten anderer deutscher Großstädte wie Gelsenkirchen (14,8\%), Duisburg (14,3\%), Essen (14,6\%), Magdeburg (9,9\%) und anderen [21]. Trotzdem ist die Generalisierbarkeit der Ergebnisse eingeschränkt, da der Versorgungsbezirk der hiesigen Klinik (Großbezirk Mitte) im regionalen Sozialbericht Berlin und Brandenburg 2017 mit 24,8\% die zweithöchste Armutsgefährdungsquote der Berliner Be-

\section{Schlussfolgerung und Fazit für die Praxis}

Mehr als die Hälfte $(55,1 \%)$ von Nutzer ${ }^{\star}$ Innen eines (teil-)stationären psychiatrischen Behandlungssettings weist Schulden, Kredite und offene Rechnungen auf, die Mehrzahl in einer Höhe von über $1000 €$. Unsere Ergebnisse legen nahe, dass mindestens $22,3 \%$ aller Befragten, die Auskunft zur Schuldenhöhe machten, eine Überschuldung aufweisen; dabei liegt die Quote deutlich höher als in der deutschen Allgemeinbevölkerung mit etwa $10 \%$.

Die regelmäßige Erfassung finanzieller Belastungen oder Schwierigkeiten in der Handhabung finanzieller Belange scheint besonders bedeutsam bei jungen Personen und Menschen mit Abhängigkeitserkrankungen.

Geeignete Unterstützungsformen sollten evaluiert werden. Gleichwohl sollten Unterstützungsmöglichkeiten hinsichtlich psychischer Erkrankungen, insbesondere eines Substanzgebrauchs, auch an Stellen sozialer Unterstützungseinrichtungen angeboten werden.

\section{Korrespondenzadresse}

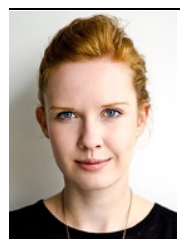

Stefanie Schreiter

Department of Psychiatry and Psychotherapy, Charité

Campus Mitte, Charité -

Universitätsmedizin Berlin, corporate member of Freie Universität Berlin, HumboldtUniversität zu Berlin, and Berlin Institute of Health Charitéplatz 1, 10117 Berlin, Deutschland stefanie.schreiter@charite.de 2018 mit 33,6\% einen vergleichsweise hohen Anteil ausländischer Personen (vgl. Gesamtberlin: 19,5\%) auf [2]. Entsprechend des Sozialberichts haben ausländische Berliner ${ }^{\star}$ Innen das höchste Armutsrisiko (28,7\%; [1]). In der hiesigen Untersuchung ergab sich kein signifikanter Unterschied im Vorliegen von Schulden, Krediten oder offenen Rechnung bei im Ausland geborener Teilnehmer*Innen; Gespräche erfolgten mit Sprach- und Kulturmittlern und ausländische Teilnehmer machten $27,3 \%$ der Teilnehmer^Innen aus.
Danksagung. Die Autor*Innen bedanken sich bei allen Teilnehmer*Innen für ihre Bereitschaft an der Studie teilzunehmen und ihre Offenheit.

Funding. Open Access funding enabled and organized by Projekt DEAL.

\section{Einhaltung ethischer Richtlinien}

Interessenkonflikt. S. Schreiter, S. Heidrich, A. Heinz, W. Rössler, R.M. Krausz, M. Schouler-Ocak, F. Bermpoh und S. Gutwinski geben an, dass kein Interessenkonflikt besteht.

Für diesen Beitrag wurden von den Autor*Innen keine Studien an Menschen oder Tieren durchgeführt. 
Für die aufgeführten Studien gelten die jeweils dort angegebenen ethischen Richtlinien.

Open Access. Dieser Artikel wird unter der Creative Commons Namensnennung 4.0 International Lizenz veröffentlicht, welche die Nutzung, Vervielfältigung Bearbeitung, Verbreitung und Wiedergabe in jeglichem Medium und Format erlaubt, sofern Sie den/die ursprünglichen Autor(en) und die Quelle ordnungsgemäß nennen, einen Link zur Creative Commons Lizenz beifügen und angeben, ob Änderungen vorgenommen wurden.

Die in diesem Artikel enthaltenen Bilder und sonstiges Drittmaterial unterliegen ebenfalls der genannten Creative Commons Lizenz, sofern sich aus der Abbildungslegende nichts anderes ergibt. Sofern das betreffende Material nicht unter der genannten Creative Commons Lizenz steht und die betreffende Handlung nicht nach gesetzlichen Vorschriften erlaubt ist, ist für die oben aufgeführten Weiterverwendungen des Materials die Einwilligung des jeweiligen Rechteinhabers einzuholen.

Weitere Details zur Lizenz entnehmen Sie bitte der Lizenzinformation auf http://creativecommons.org/ licenses/by/4.0/deed.de.

\section{Literatur}

1. Amt für Statistik Berlin-Brandenburg (2018) Regionaler Sozialbericht Berlin und Brandenburg 2017. Amt für Statistik Berlin-Brandenburg, Potsdam

2. Amt für Statistik Berlin-Brandenburg (2018) Statistischer Bericht; Einwohnerinnen und Einwohner im Land Berlin am 30. Juni 2018 https://doi.org/10. 1055/s-0029-1194251

3. Baker E, Lester L, Mason K, Bentley R (2020) Mental health and prolonged exposure to unaffordable housing: a longitudinal analysis. Soc Psychiatry Psychiatr Epidemiol 55:715-721. https://doi.org/ 10.1007/s00127-020-01849-1

4. Drentea P, Reynolds JR (2012) Neither a borrower nor a lender be: the relative importance of debt and SES for mental health among older adults. J Aging Health 24:673-695. https://doi.org/10. $1177 / 0898264311431304$

5. European Commission (2008) Towards a common operational European definition over-indebtedness. http://www.oee.fr/files/ study_overindebtedness_en.pdf. Zugegriffen: 01.07.2020

6. Graubner B, Auhuber T (2013) ICD-10-GM (internationale statistische Klassifikationen der Krankheiten und verwandter Gesundheitsprobleme). Deutscher Ärzteverlag,

7. Hintikka J, Viinamäki $H$, Tanskanen A et al (1998) Suicidal ideation and parasuicide in the Finnish general population. Acta Psychiatr Scand 98:23-27. https://doi.org/10.1111/j.1600-0447. 1998.tb10037.x

8. Holmgren R, Nilsson Sundström $E$, Levinsson $H$, Ahlström R (2019) Coping and financial strain as predictors of mental illness in over- indebted individuals in Sweden. Scand J Psychol 60:50-58. https://doi.org/10.1111/sjop.12511

9. Jenkins R, Bhugra D, Bebbington P et al (2008) Debt, income and mental disorder in the general population. Psychol Med 38:1485-1493. https:// doi.org/10.1017/S0033291707002516
10. Jenkins R, Fitch C, Hurlston M, Walker F (2009) Recession, debt and mental health: challenges and solutions. Ment Health Fam Med 6:85-90

11. Konrad M (2018) Das Bundesteilhabegesetz als Chancefür einevernetztegemeindepsychiatrische Versorgung. Psychiatr Prax 45:229-232. https:// doi.org/10.1055/a-0623-8871

12. Meltzer $H$, Bebbington $P$, Brugha $T$ et al (2011) Personal debt and suicidal ideation. Psychol Med 41:771-778. https://doi.org/10. 1017/S0033291710001261

13. Meltzer $\mathrm{H}$, Bebbington $\mathrm{P}$, Brugha T et al (2013) The relationship between personal debt and specific common mental disorders. Eur J Public Health 23:108-113. https://doi.org/10.1093/eurpub/ cks021

14. Patel V, Pereira J, Coutinho L et al (1998) Poverty , psychological disorder and disability in primary care attenders in Goa, India. Br J Psychiatry 172:533-536

15. Pothen M, Kuruvilla A, PhilipKetal (2003)Common mental disorders among primary care attenders in Vellore, South India: nature, prevalence and risk factors. Int J Soc Psychiatry 49:119-125

16. Richardson T, Elliott P, Roberts R (2013) The relationship between personal unsecured debt and mental and physical health: a systematic review and meta-analysis. Clin Psychol Rev 33:1148-1162. https://doi.org/10.1016/j.cpr.2013.08.009

17. Schreiter S, Heidrich S, Zulauf J et al (2019) Housing situation and health care for patients in a psychiatric centre in Berlin, Germany-a crosssectional patient survey. BMJ Open. https://doi. org/10.1136/bmjopen-2019-032576

18. Statista Anzahl der überschuldeten Privatpersonen* in Deutschland von 2004 bis 2019. https:// de.statista.com/statistik/daten/studie/166338/ umfrage/anzahl-der-schuldner-in-deutschlandseit-2004/.Zugegriffen:18. Nov. 2019

19. Statistisches Bundesamt Pressemitteilung Nr. 199 vom 28. Mai 2019: Private Überschuldung: Starke Unterschiede zwischen Jung und Alt. https:// www.destatis.de/DE/Presse/Pressemitteilungen/ 2019/05/PD19_199_635.html. Zugegriffen: 18. Nov. 2019

20. IBM Corp (2010) IBM SPSS statistics for Windows, version 19.0. IBM Corp, Armonk, NY

21. Bundesagentur für Arbeit Arbeitslosenstatistik. https://statistik.arbeitsagentur.de/ Navigation/Statistik/Statistik-nach-Regionen/ BA-Gebietsstruktur-Nav.html. Zugegriffen: 25 Juni 2019

\section{MEditorial
Manager}

\section{Hilfestellungen für den Editorial Manager}

Das Einreichungs- und Begutachtungssystem Ihrer Zeitschrift

Sowohl für die ganz alltäglichen Fragen in der Handhabung des Editorial Managers als auch für spezielle Problematiken finden Sie auf www.springermedizin.de/editorialmanager eine Vielzahl an Handreichungen, die Ihnen die Arbeit als Gutachter*in, Autor*in oder Herausgeber*in erleichtern.

Über Videos, einseitige Schritt-für-SchrittAnleitungen oder ein umfangreiches Manual werden Sie durch die einzelnen Punkte geführt, wie:

Wie reiche ich ein Manuskript ein?

- Wie finde ich passende Gutachter*innen?

- Wie lade ich Gutachter*innen ein?

- Wie nehme ich ein Gutachten an bzw. lehne es ab?

- Wo erkenne ich, in welchem Status ein Mansukript ist?

- Wie ändere ich meine persönlichen Informationen?

- Wo kann ich meinen Urlaub eintragen?
Zugang auch über QR-Code:

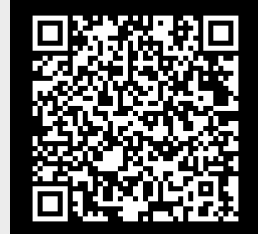

